\title{
Crisis management during anaesthesia: bronchospasm
}

\author{
R N Westhorpe, G L Ludbrook, S C Helps
}

Qual Saf Health Care 2005;14:e7 (http://www.qshc.com/cgi/content/full/14/3/e7). doi: 10.1136/qshc.2002.004457

Background: Bronchospasm in association with anaesthesia may appear as an entity in its own right or be a component of another problem such as anaphylaxis. It may present with expiratory wheeze, prolonged exhalation or, in severe cases, complete silence on auscultation.

Objectives: To examine the role of a previously described core algorithm "COVER ABCD-A SWIFT $\mathrm{CHECK}^{\prime \prime}$, supplemented by a specific sub-algorithm for bronchospasm, in the diagnosis and management of bronchospasm occurring in association with anaesthesia.

Methods: The potential performance of this structured approach for each of the relevant incidents among the first 4000 reported to the Australian Incident Monitoring Study (AIMS) was compared with the actual management as reported by anaesthetists involved.

See end of article for authors' affiliations Results: There were 103 relevant incidents among the first 4000 AIMS reports, 22 of which were associated with allergy or anaphylaxis. Common presenting signs, in addition to wheeze, were decreased pulmonary compliance and falling oxygen saturation. Of the non-allergy/anaphylaxis related incidents, ......................

Correspondence to: Professor W B Runciman, President, Australian Patient Safety Foundation, GPO Box 400, Adelaide, South Australia 5001, Australia; research@apsf. net.au $80 \%$ occurred during induction or maintenance of anaesthesia. Of these, the principal causes of bronchospasm were airway irritation (35\%), problems with the endotracheal tube (23\%), and aspiration of gastric contents (14\%). It was considered that, properly used, the structured approach recommended would have led to earlier recognition and/or better management of the problem in $10 \%$ of cases, and would not have harmed any patient had it been applied in all of them.

Conclusion: Bronchospasm may present in a variety of ways and may be associated with other life threatening conditions. Although most cases are handled appropriately by the attending anaesthetist, the use of a structured approach to its diagnosis and management would lead to earlier recognition and/or Accepted 1 January 2005 better management in $10 \%$ of cases.

B ronchospasm usually manifests during anaesthesia as an expiratory wheeze, prolonged expiration and/or increased inflation pressures during intermittent positive pressure ventilation (IPPV). Wheeze may be audible either with or without auscultation, but can only be present if there is gas flow in the patient's airways. Thus, in cases of severe bronchospasm, the chest may be silent on auscultation and the diagnosis may rest on correct assessment of increased inflation pressures.

Bronchospasm may appear as an entity in its own right or be a component of another problem such as anaphylaxis (which is dealt with elsewhere in this series of articles ${ }^{1}$ ) and is usually triggered by some manoeuvre, often in patients with a pre-existing airway disease such as asthma.

Wheeze may occur not only as a result of bronchospasm but may be heard with misplacement of the endotracheal tube (in the oesophagus or a bronchus, for example) and with pulmonary oedema or adult respiratory distress syndrome (ARDS). Increased inspiratory pressures may occur not only with any of these conditions but with obstruction of the natural or an artificial airway or of any component of the breathing circuit (including any respiratory filters) and decreased compliance of the lung (for example, with atelectasis) or chest (for example, haemopneumothorax, fentanyl induced rigidity). It was therefore decided to examine the role of a structured approach to the diagnosis and management of bronchospasm or wheeze in association with anaesthesia.

In 1993 a "core" crisis management algorithm, represented by the mnemonic COVER ABCD-A SWIFT CHECK (the AB precedes COVER for the non-intubated patient), was proposed as the basis for a systematic approach to any crisis during anaesthesia where it is not immediately obvious what should be done or where actions taken have failed to remedy the situation. ${ }^{2}$ This was validated against the first 2000 incidents reported to the Australian Incident Monitoring Study (AIMS). AIMS is an ongoing study which involves the voluntary anonymous reporting of any unintended incident which reduced or could have reduced the safety margin for a patient. $^{3}$

It was concluded that, if this algorithm had been correctly applied, a functional diagnosis would have been reached within 40-60 seconds in 99\% of applicable incidents and that the learned sequence of actions recommended by the COVER portion would have led to appropriate steps being taken to handle the $60 \%$ of problems relevant to this portion of the algorithm. ${ }^{2}$ However, this study also showed that the $40 \%$ of problems represented by the remainder of the algorithm, ABCD-A SWIFT CHECK, were not always promptly diagnosed or appropriately managed. ${ }^{2-4}$ It was decided that it would be useful, for these problems, to develop a set of subalgorithms in an easy-to-use crisis management manual. ${ }^{5}$ This study reports on the potential place of the COVER ABCD-A SWIFT CHECK algorithm in the diagnosis and initial management of bronchospasm, provides an outline of a specific crisis management sub-algorithm for bronchospasm, and provides an indication of the potential value of using this structured approach.

\section{METHODS}

Of the first 4000 incidents reported to AIMS, those which made reference to bronchospasm or wheeze were extracted and analysed for relevance, presenting features, type of surgery, cause, management, and outcome. The COVER ABCD-A SWIFT CHECK algorithm, described elsewhere in this series of articles, ${ }^{5}$ was applied to each relevant report to determine the stages at which the problem might have been diagnosed and to confirm that activating the COVER portion 


\section{BRONCHOSPASM}

\section{SIGNS (1)*}

Increasing circuit pressure

Desaturation

Wheeze (auscultate)

Rising $\mathrm{ETCO}_{2}$ and prolonged expiration

Reduction in tidal volumes

\section{THINK OF (2)}

Anaphylaxis/allergy to drugs/IV fluids/latex

Airway manipulation/irritation/secretions/soiling

Oesophageal/endobronchial intubation

Pneumothorax

Inadequate anaesthetic depth or failure of anaesthetic delivery system

\section{EMERGENCY MANAGEMENT}

\section{$100 \%$ Oxygen}

Cease stimulation/surgery

Request immediate assistance

Deepen anaesthesia (3)

If intubated exclude endobronchial or oesophageal position (4)

If mask/LMA in use consider early:

laryngospasm/airway obstruction $\rightarrow$ pages $12 \& 14^{* *}$

regurgitation/vomit/aspiration $\rightarrow$ page $16^{* *}(5)$

Give adrenaline or salbutamol (see over)

If you cannot ventilate via an ETT consider:

Misplaced/kinked/blocked ETT or circuit $\rightarrow$ page $7^{* *}$

Pneumothorax $\rightarrow$ page $46^{* *}$

Aspiration $\rightarrow$ page $16^{* *}$

Anaphylaxis $\rightarrow$ page $48^{* *}$

Pulmonary oedema $\rightarrow$ page $24^{* *}$

Consider possible obstruction distal to ETT

Try pushing a small tube past it, or push the obstruction down one bronchus and ventilate the other lung.

CONSIDER ANAPHYLAXIS $\rightarrow$ page $48^{* *}$

CONSIDER PULMONARY OEDEMA $\rightarrow$ page $24^{* *}$

The sub-algorithm forms a facing page of the

Crisis Management Manual6.

* Numbers in brackets refer to Notes in the right hand panel.

** Page references refer to Crisis Management Manual ${ }^{6}$.
FURTHER CARE:

Depends on patient's condition, and cause

Bronchodilators as necessary

Chest X-ray

Admit to $\mathrm{HDU} / \mathrm{ICU}$ if necessary

\section{NOTES:}

(1) $30 \%$ of 103 relevant incidents described increased peak inflation pressures, and a further $31 \%$ described "bronchospasm"/wheeze as the initial sign. $21 \%$ reported desaturation as the first sign.

$3 \%$ reported rising $\mathrm{ETCO}_{2}$ and $1 \%$ revealed a flat capnogram indicating no gas flow.

(2) Allergy/anaphylaxis - 22/103 (21\%) of incidents.

Of the remaining 81 cases of bronchospasm:

$44 \%$ occurred at induction and of these:

$64 \%$ were due to airway irritation

$17 \%$ were due to ETT misplacement

$11 \%$ were due to aspiration

$8 \%$ were due to other causes.

$36 \%$ occurred during maintenance and of these:

$31 \%$ were due to ETT problem

$14 \%$ were due to aspiration with an LMA.

$20 \%$ occurred during emergence/recovery and of these:

$38 \%$ had no specific cause identified

$25 \%$ were due to pulmonary oedema

$18 \%$ were due to aspiration.

(3) $55 \%$ of cases of bronchospasm at induction were idiopathic or presumed to be due to airway irritation from laryngoscopy and/or intubation.

(4) $12 \%$ were associated with oesophageal intubation and $2.5 \%$ with endobronchial intubation.

(5) $12 \%$ of cases were associated with aspiration.

Recommended dosages:

Salbutamol $0.5 \% 1 \mathrm{ml}(5 \mathrm{mg})$ solution nebulised for adult

or aerosol puffer, 2 puffs $(0.1 \mathrm{mg} /$ puff)

or $0.5 \% 0.1 \mathrm{ml}$ in $1 \mathrm{ml}$, injected down ETT $(0.5 \mathrm{mg}$ adult).

Adrenaline $0.001 \mathrm{mg} / \mathrm{kg}$ bolus $(0.01 \mathrm{ml} / \mathrm{kg}$ of $1: 10000 \mathrm{soln}$.)

Repeat bolus, or commence infusion $0.00015 \mathrm{mg} / \mathrm{kg} / \mathrm{min}$

Adrenaline; easy dosing for adults, see page $71^{*}$

Titrate to heart rate, blood pressure, and bronchodilator effect.

These notes comprise a reverse side of a page of the

Crisis Management Manual6.

* Page references refer to the Crisis Management Manual 6 .

Figure 1 Bronchospasm.

would have led to appropriate initial steps being taken. As bronchospasm is not dealt with by this algorithm, a specific sub-algorithm was developed for this problem (see fig 1), and its putative effectiveness was tested against the reports. How this was done is described elsewhere in this series of articles. ${ }^{5}$ The potential value of this structured approach - that is, the application of COVER ABCD-A SWIFT CHECK to the diagnosis and initial management of this problem followed by the application of the sub-algorithm for bronchospasmwas assessed in the light of the AIMS reports by comparing its potential effectiveness for each incident with that of the actual management, as recorded in each report.

\section{RESULTS}

Of the first 4000 AIMS reports, 174 contained the words "wheeze" or "bronchospasm". Of these, 71 were excluded from further analysis. Reasons for exclusion were those reports in which bronchospasm or wheeze were specifically excluded by the reporter or mentioned as not being present (59 reports); a further nine reports in which there were insufficient details to confirm a diagnosis or where bronchospasm occurred during a regional anaesthetic or before induction; and three reports where mild bronchospasm or wheeze occurred as part of another condition for which an AIMS report was generated, and the bronchospasm did not require specific treatment.

The remaining 103 reports were analysed as follows:

\section{Stage of anaesthesia}

The stage of anaesthesia-that is, induction, maintenance, emergence or recovery-at which bronchospasm or wheeze occurred is shown in table 1.

\section{Induction}

The causes of bronchospasm and/or wheeze during the induction phase of anaesthesia are summarised in table 2 . Of the total 42 reports, 23 were idiopathic or presumed to be due to airway irritation from laryngoscopy and/or intubation; six were related to anaphylaxis or an anaphylactoid reaction including one due to massive histamine release after 
Table 1 Stage of anaesthesia at which bronchospasm or wheeze was detected

\begin{tabular}{lcc}
\hline $\begin{array}{l}\text { Stage of } \\
\text { anaesthesia }\end{array}$ & $\begin{array}{l}\text { Bronchospasm due } \\
\text { to any cause }\end{array}$ & $\begin{array}{l}\text { Bronchospasm due to } \\
\text { allergy/anaphylaxis }\end{array}$ \\
\hline $\begin{array}{l}\text { Induction } \\
\text { Maintenance }\end{array}$ & 42 & 6 \\
Emergence & 44 & 15 \\
Recovery & 7 & 0 \\
Total & 10 & 1 \\
\hline
\end{tabular}

morphine administration; six were related to misplacement of the endotracheal tube; and four were caused by aspiration of regurgitated gastric contents. There was one report of bronchospasm associated with pulmonary oedema following a failed intubation and two reports where the cause of bronchospasm was unknown but was thought to be due to allergy.

\section{Bronchospasm presumed due to airway irritation on induction}

Of the 23 reports where there was no other cause, 16 concerned patients who may be considered to be predisposed to bronchospasm from airway irritation. They included those with pre-anaesthetic histories of asthma, chronic or acute bronchitis, chronic obstructive airway disease (COAD), reflux oesophagitis, or heavy smoking. Some patients had histories which included more than one of the predisposing factors and several had not volunteered the information when questioned by the anaesthetist preoperatively. One report described bronchospasm occurring after a difficult intubation, presumably also due to irritation of the airway; one mentioned wheeze occurring in association with resolution of laryngospasm on induction in a child; and one described bronchospasm in association with laryngospasm resulting from traumatic insertion of a laryngeal mask airway.

\section{Bronchospasm associated with misplacement of the endotracheal tube}

Six reports mentioned problems of intubation in association with bronchospasm. Five occurred with oesophageal intubation; however, bronchospasm was described as being present during oesophageal intubation in only two cases, the other three developing bronchospasm after the endotracheal tube was correctly placed. There was one report of bronchospasm with endobronchial intubation.

\section{Maintenance}

There were 44 reports of bronchospasm and/or wheeze in association with the maintenance phase of anaesthesia (table 3). Of these, 15 were related to instances of
Table 3 Bronchospasm or wheeze occurring during the maintenance phase of anaesthesia

\begin{tabular}{lr}
\hline Cause & No \\
\hline Anaphylaxis (or severe allergy) & 15 \\
Bronchospasm (no defined cause) & 9 \\
Endotracheal tube or ventilator problem & 10 \\
Aspiration associated with LMA & 4 \\
Aspiration not associated with LMA & 1 \\
Pneumothorax & 1 \\
Pulmonary oedema & 1 \\
Profuse bronchial mucus & 1 \\
Drug induced & 2 \\
Total & 44 \\
\hline LMA, laryngeal mask airway. &
\end{tabular}

anaphylaxis (or severe allergy), nine were due to bronchospasm of no defined cause, five were due to endobronchial intubation, one was due to pulmonary oedema, and one was due to profuse bronchial mucus in a heavy smoker.

\section{Bronchospasm presumed due to airway irritation}

There were nine reports where there was no defined cause, although five of these occurred in patients who were noted to have one or more of the following predisposing factors: heavy smoking, asthma or bronchitis.

\section{Endotracheal tube and ventilator problems}

Five of these reports cited endobronchial intubation as the precipitating cause of bronchospasm.

\section{Aspiration}

There were five reports of pulmonary aspiration during maintenance of anaesthesia. Four were associated with the use of the laryngeal mask. In three of these reports the aspiration was of regurgitated gastric contents, and one was aspiration of thick pharyngeal mucus in a child. There was one report of aspiration after intubation with a non-cuffed endotracheal tube in a child.

\section{Drug induced bronchospasm}

Rapid injection of vancomycin was noted as the cause in one report, treatment being directed at the histamine release by the injection of salbutamol, metaraminol and fluids. One report described bronchospasm after protamine injection, with the signs settling in a few minutes without treatment.

\section{Pneumothorax}

There was one case of bronchospasm associated with pneumothorax in an elderly patient undergoing a difficult bronchoscopy using a Venturi ventilation technique.

Table 4 Bronchospasm or wheeze occurring during the emergence or recovery phase of anaesthesia

Table 2 Causes of bronchospasm or wheeze during the induction phase of anaesthesia

\begin{tabular}{lc}
\hline Cause & No \\
\hline Bronchospasm presumed due to airway & 23 \\
irritation & \\
Anaphylaxis & 6 \\
Misplacement of endotracheal tube & 6 \\
Aspiration of gastric contents & 4 \\
Pulmonary oedema (following & 1 \\
failed intubation) & 2 \\
Unknown, possibly allergy & 42 \\
Total & \\
\hline
\end{tabular}




\section{Emergence and recovery}

There were 17 reports of bronchospasm and/or wheeze during the emergence or recovery phases of anaesthesia, seven during emergence and 10 during recovery. The results are summarised in table 4. All of the reports of bronchospasm with no defined cause occurred in patients who exhibited predisposing factors including asthma, a history of heavy smoking, or chronic obstructive airways disease.

Three of the cases of pulmonary oedema were initially treated as bronchospasm. The fourth case of pulmonary oedema was recognised as being due to rapid infusion of colloid in an elderly patient. The patient in whom bronchospasm was reported to have occurred upon extubation was managed as for laryngospasm with the appropriate outcome and, as such, the diagnosis of bronchospasm may be questioned. One patient developed unilateral bronchospasm and pulmonary oedema which resolved over several days and for which no cause could be found.

\section{Presenting signs}

Bronchospasm associated with anaphylaxis or allergy

There were 22 reports of anaphylaxis or severe allergy in which bronchospasm was included in the signs exhibited by the patient. The range of presenting signs is shown in table 5. The appearance of a rash was the first sign of anaphylaxis in six of the 22 reports, but wheeze, bronchospasm or increased pulmonary inflation pressures-when appearing together with hypotension-were often followed by the appearance of a rash. A rash or cutaneous flush was eventually present in 16 of the 22 reports of anaphylaxis or severe allergy.

\section{Bronchospasm not associated with anaphylaxis or severe allergy}

All reports apart from those where anaphylaxis or severe allergy was the cause of bronchospasm were analysed to determine which sign first alerted the anaesthetist to the presence of a problem (table 6).

Of the 81 reports examined, 27 first indicated a problem by the need for high inflation pressures; 23 reports described "bronchospasm" as being the first sign, although the narrative description does not indicate whether this was wheeze or high inflation pressure; and five described wheeze as the first sign. Thus, it may be concluded that, in 55 of the 81 reports $(68 \%)$, wheeze or increased inflation pressure was the sign which triggered the diagnostic and management process.

Low oxygen saturation as shown by the pulse oximeter was the first sign of a problem in 19 reports. A change in the capnogram was the first sign in three reports, two showing a high end-tidal $\mathrm{CO}_{2}$ and one where the capnogram fell to the baseline indicating no gas flow associated with very severe bronchospasm. There were three reports of low tidal volume

Table 5 First presenting sign of anaphylaxis or severe allergy associated with bronchospasm in all phases of anaesthesia

\begin{tabular}{lc}
\hline $\begin{array}{l}\text { First presenting sign of anaphylaxis or } \\
\text { severe allergy }\end{array}$ & No \\
\hline Rash & 6 \\
Increased inflation pressure & 4 \\
Wheeze & 4 \\
Hypotension & 4 \\
Falling oxygen saturation & 3 \\
Capnography change with LMA & 1 \\
Total & 22 \\
\hline Note that 16 of the 22 cases eventually exhibited a rash.
\end{tabular}

as the first sign of bronchospasm and one report where hypotension led to the diagnosis.

\section{DISCUSSION}

\section{Induction and maintenance}

When the COVER ABCD "core" algorithm was applied to the 103 reports as analysed, it was apparent that the basic problem should have been at least detected and diagnosed by the completion of " $\mathrm{B}$ " in all cases. Diagnosis of pneumothorax may have proved somewhat obscure, but the " $\mathrm{B}$ " stage of the algorithm asks that it be considered as a possibility. The likely diagnostic stages of the COVER ABCD algorithm are as follows:

C, Colour: Observed desaturation or cyanosis, confirmed by pulse oximetry. Rash or other cutaneous manifestation of allergy.

Capnography: rising end-tidal $\mathrm{CO}_{2}$ being a diagnostic sign of hypoventilation from any cause. Very severe bronchospasm may be accompanied by a fall in end-tidal $\mathrm{CO}_{2}$ to zero.

V2, Ventilate by hand: observe and auscultate. This is the level of the core algorithm from which the sub-algorithm for bronchospasm is most likely to stem.

E1, Check endotracheal tube: This stage will diagnose and, in most cases, provide information for correct management of endotracheal tube problems such as oesophageal intubation, endobronchial intubation, and a kinked or obstructed tube. Importantly, if a laryngeal mask airway is in use, the pharynx should be inspected for regurgitation and potential aspiration.

E2, Ventilate with an alternative system: such as a self-inflating resuscitation bag. This will eliminate all causes of high circuit pressure which are equipment related and is an important diagnostic step, even if the "bronchospasm" sub-algorithm has been commenced.

Although desaturation is a signal that a crisis is evolving and is thus a trigger for proceeding with a process for diagnosis and treatment of the crisis in anaesthesia, it would appear that it is not a good discriminator for detecting bronchospasm. However, changes in oxygen saturation are likely to be useful as a guide to the efficacy of management.

\section{Emergence and recovery}

Although high lung inflation pressures may be detected at the time of emergence and should be dealt with appropriately by the bronchospasm sub-algorithm, other detection of bronchospasm during emergence and recovery will depend on the appearance of wheeze, audible either with or without auscultation. In these cases, and especially during the postoperative recovery phase, care must be exercised to eliminate pulmonary oedema or aspiration as a cause of wheeze.

\section{Diagnosis and treatment of bronchospasm}

A suggested sub-algorithm for the management of bronchospasm is detailed in fig 1 . The sub-algorithm was applied to all reports not involving anaphylaxis or severe allergy (that is, to 81 reports). The authors consider that the sub-algorithm would have performed satisfactorily in all cases and, in 73 cases $(90 \%)$, it would have performed as well as the clinician as described in the report narrative. In eight cases $(10 \%)$ the authors consider that the sub-algorithm would have performed better than the clinical management as described. In five of these cases no treatment was administered when it is considered that earlier resolution may have been achieved by the use of bronchodilator therapy. In two cases bronchodilator therapy would have been instituted at an earlier stage and in one case the delayed diagnosis could have been avoided. 
Table 6 Presenting sign of bronchospasm (excluding cases of anaphylaxis/severe allergy) during induction (I), maintenance (M), and emergency/recovery (E\&R)

\begin{tabular}{lrrrr}
\hline Presenting sign & I & M & E\&R & Total \\
\hline Increased inflation pressure & 19 & 8 & 0 & 27 \\
Low oxygen saturation & 3 & 8 & 8 & 19 \\
"Bronchospasm" & 9 & 8 & 6 & 23 \\
Wheeze & 3 & 1 & 1 & 5 \\
Change in capnogram & 2 & 1 & 0 & 3 \\
Hypoventilation (low tidal volume) & 0 & 2 & 1 & 3 \\
Hypotension & 0 & 1 & 0 & 1 \\
Total & 36 & 29 & 16 & 81 \\
\hline
\end{tabular}

In recognising and managing bronchospasm due to various causes, diagnostic signs are likely to include increased inflation pressure, wheeze, falling oxygen saturation, and rising end-tidal $\mathrm{CO}_{2}$ concentration. The appearance of a rash or hypotension will point to a diagnosis of anaphylaxis.

In a number of reports of bronchospasm, intravenous salbutamol was used instead of nebulised or atomised administration into the endotracheal tube or breathing circuit. In all but one case this appeared to delay resolution of the problem. Some reports also alluded to difficulty in applying nebulisers to circuits. A readily available method of

\section{Key messages}

- From the first 4000 incidents reported to AIMS, 103 reports of bronchospasm (3\%) were subjected to analysis.

- Twenty two of the 103 reports were associated with allergy or anaphylaxis.

- The most common first presenting signs of anaphylaxis or severe allergy in this series were a rash (6 cases), increased inflation pressure (4 cases), wheeze (4 cases), and hypotension (4 cases).

- Overall, in the 81 reports examined lexcluding anaphylaxis/severe allergy), 27 reported the need for high inflation pressures as the first presenting sign of bronchospasm.

- Eighty six of the 103 bronchospasm reports (83\%) occurred during either the induction or maintenance phase of anaesthesia.

- Airway irritation accounted for half of the induction occurrences.

- Anaphylaxis or an endotracheal tube or ventilator problem accounted for more than half of the occurrences during the maintenance phase.

- Of the 17 occurrences of bronchospasm during emergence or recovery, six had no defined cause and another four were due to pulmonary oedema.

- Desaturation alone is not a good discriminator for detecting bronchospasm, but saturation changes are a useful guide during management.

- The specific bronchospasm sub-algorithm was applied to 81 reports (anaphylaxis/severe allergy cases dealt with in another paper in this series were excluded). In $90 \%$ the sub-algorithm was potentially as good as the clinician's reported management and in 10\% was potentially superior to the clinical management described. introducing salbutamol spray to the breathing circuit is advantageous in the management of bronchospasm.

The following features are considered to be important in the treatment of bronchospasm (fig 1):

- Consider steps E1 and E2 of the COVER ABCD core algorithm.

- If laryngeal mask airway (LMA) is in use, aspiration as the cause of bronchospasm should be considered early.

- Think of anaphylaxis or pulmonary oedema as alternative diagnoses.

- Review the patient's history, especially that which relates to respiratory disease.

- Review the anaesthetic for any drugs which may have given rise to bronchospasm.

- If the patient fails to respond to treatment as expected, think of the possibility of pulmonary oedema or pneumothorax.

- If wheeze or increased pulmonary inflation pressures are accompanied by hypotension, consider the possibility of anaphylaxis.

\section{CONCLUSION}

Perioperative bronchospasm may range in presentation from complete silence on auscultation or a few quiet musical notes at the end of exhalation to loud discordant expiratory noise. Accompanying diagnostic signs may include a rise in pulmonary inflation pressure, falling oxygen saturation, and a change in the slope of the end-tidal $\mathrm{CO}_{2}$ trace. As such, these signs may be indicative of mild reactive airways disease, oesophageal intubation, or a catastrophic anaphylactic reaction. Anaesthetists must therefore understand the precipitating factors, recognise the presenting signs, and be prepared to undertake a logical plan of management. The plan should not only treat the problem and its pathophysiological consequences, but should also prompt the anaesthetist to re-review the patient's evolving condition.

Finally, it is important that a full explanation of what happened be given to the patient, that the problem be clearly documented in the anaesthetic record, and that the patient be given a letter to warn future anaesthetists. If a particular precipitating event was significant or a particular action was useful in resolving the crisis, this should be clearly explained and documented.

\section{ACKNOWLEDGEMENTS}

The authors would like to thank all the anaesthetists in Australia and New Zealand who contributed to the 4000 incident reports upon which this and the other 24 papers in the Crisis Management Series are based. The coordinators of the project also thank Liz Brown for preparing the draft of the original Crisis Management Manual; Loretta Smyth for typing; Monika Bullock RN for earlier coding and classifying of data; Dr Charles Bradfield for the electronic version of 
the algorithms; Dr Klee Benveniste for literature research; and Drs Klee Benveniste, Michal Kluger, John Williamson and Andrew Paix for editing and checking manuscripts.

\section{Authors' affiliations}

R N Westhorpe, Deputy Director, Department of Paediatric Anaesthesia and Pain Management, Royal Children's Hospital, Parkville, Victoria, Australia

G L Ludbrook, Professor, Department of Anaesthesia and Intensive Care, University of Adelaide and Royal Adelaide Hospital, Adelaide, South Australia, Australia

S C Helps, Department of Medical Biochemistry, School of Medicine, Flinders University, Bedford Park, South Australia, Australia

This study was coordinated by the Australian Patient Safety Foundation, GPO Box 400, Adelaide, South Australia 5001, Australia.

\section{REFERENCES}

1 Currie $M$, Kerridge R, Bacon AK, et al. Crisis management during anaesthesia: anaphylaxis and allergy. Qual Saf Health Care 2005;14:e19.

2 Runciman WB, Webb RK, Klepper ID, et al. Crisis management: validation of an algorithm by analysis of 2000 incident reports. Anaesth Intensive Care 1993:21:579-92.

3 Webb RK, Currie M, Morgan CA, et al. The Australian Incident Monitoring Study: an analysis of 2000 incident reports. Anaesth Intensive Care 1993;21:520-8.

4 Webb RK, van der Walt JH, Runciman WB, et al. Which monitor? An analysis of 2000 incident reports. Anaesth Intensive Care 1993;21:529-42.

5 Runciman WB, Kluger MT, Morris RW, et al. Crisis management during anaesthesia: the development of an anaesthetic crisis management manual. Qual Saf Health Care 2005; 14:e1.

6 Australian Patient Safety Foundation. Crisis Management Manual: COVER $A B C D$ A SWIFT CHECK. Adelaide: Australian Patient Safety Foundation, 1996, 74 pp. Available at http://www.apsf.net.av/anaesthesia.htm (accessed 6 September 2004). 